\title{
Spin accumulation in diffusive conductors with Rashba and Dresselhaus spin-orbit interaction
}

\author{
Mathias Duckheim* and Daniel Loss \\ Department of Physics, University of Basel, CH-4056 Basel, Switzerland \\ Matthias Scheid ${ }^{\dagger}$ and Klaus Richter \\ Institut für Theoretische Physik, Universität Regensburg, D-93040 Regensburg, Germany \\ İnanç Adagideli \\ Faculty of Engineering and Natural Sciences, Sabanci University, Tuzla, Istanbul 34956, Turkey \\ Philippe Jacquod \\ Department of Physics, University of Arizona, 1118 E. 4th Street, Tucson, Arizona 85721, USA \\ (Received 23 September 2009; revised manuscript received 23 December 2009; published 2 February 2010)

\begin{abstract}
We calculate the electrically induced spin accumulation in diffusive systems due to both Rashba (with strength $\alpha$ ) and Dresselhaus (with strength $\beta$ ) spin-orbit interaction. Using a diffusion equation approach we find that magnetoelectric effects disappear and that there is thus no spin accumulation when both interactions have the same strength, $\alpha= \pm \beta$. In thermodynamically large systems, the finite spin accumulation predicted by Chaplik, Entin, and Magarill [Physica E 13, 744 (2002)] and by Trushin and Schliemann [Phys. Rev. B 75, 155323 (2007)] is recovered an infinitesimally small distance away from the singular point $\alpha= \pm \beta$. We show however that the singularity is broadened and that the suppression of spin accumulation becomes physically relevant (i) in finite-sized systems of size $L$, (ii) in the presence of a cubic Dresselhaus interaction of strength $\gamma$, or (iii) for finite-frequency measurements. We obtain the parametric range over which the magnetoelectric effect is suppressed in these three instances as (i) $|\alpha|-|\beta| \lesssim 1 / m L$, (ii) $|\alpha|-|\beta| \lesssim \gamma p_{\mathrm{F}}^{2}$, and (iii) $|\alpha|-|\beta|$ $\lesssim \sqrt{\omega / m p_{\mathrm{F}} \ell}$ with $\ell$ the elastic mean-free path and $p_{\mathrm{F}}$ the Fermi momentum. We attribute the absence of spin accumulation close to $\alpha= \pm \beta$ to the underlying $\mathrm{U}(1)$ symmetry. We illustrate and confirm our predictions numerically.
\end{abstract}

DOI: 10.1103/PhysRevB.81.085303 PACS number(s): 73.23. - b, 72.25.Dc, 75.80.+q, 85.75. $-\mathrm{d}$

\section{INTRODUCTION}

Many recent works have explored mechanisms to generate spin accumulations and spin currents by passing electric currents through spin-orbit-coupled electronic systems. On the theoretical side, two related mechanisms have been proposed in disordered metals as alternatives to spin injection via ferromagnets or by optical means. They are currentinduced transverse spin currents or voltages, a.k.a. the spinHall effect, ${ }^{1-12}$ and current-induced spin polarization (CISP). ${ }^{13-18}$ The interplay between the two effects has been investigated in Ref. 19. These effects have been to some extent demonstrated experimentally, ${ }^{20-24}$ and recent theoretical works have extended them to include the mesoscopic regime, where fluctuations of both longitudinal and transverse spin currents in mesoscopic ballistic and diffusive systems are being investigated. ${ }^{25-29}$ Most remarkably, it has been found that the same universality that applies to mesoscopic charge transport ${ }^{30}$ also applies to mesoscopic spin transport. ${ }^{25}$

The main focus of these theoretical as well as experimental efforts is to use spin-orbit interactions (SOIs) as a way to couple external electric fields to electronic spins, the hope being to generate, manipulate, and/or measure spin currents and accumulations by purely electrical means. SOI, however, also has the undesired effect of randomizing electronic spins. ${ }^{31}$ This dichotomy theoretically limits the use of SOIbased magnetoelectric devices as components of information processors to the regime where the size of the device is much less than the spin-relaxation length. A way to increase the spin-relaxation length has been proposed in Ref. 32 for systems which exhibit SOI of both the Rashba, ${ }^{33}$

$$
H_{\mathrm{R}}=\alpha\left(p_{x} \sigma_{y}-p_{y} \sigma_{x}\right)
$$

and the Dresselhaus type ${ }^{34}$

$$
H_{\mathrm{D}}=\beta\left(p_{x} \sigma_{x}-p_{y} \sigma_{y}\right),
$$

where $\sigma_{x, y}$ are Pauli matrices. When the two interactions have equal strength, $\alpha= \pm \beta$, the SOI rotates electron spins around a single fixed axis. The spin along this axis becomes conserved while spins along the perpendicular directions undergo a deterministic rotation that depends only on the starting and end points of their trajectory. In particular, spins are not rotated along closed trajectories, therefore mesoscopic systems exhibit negative magnetoresistance when $\alpha= \pm \beta,{ }^{35-37}$ i.e., weak localization and not weak antilocalization, just as if SOI were absent. An effective spin randomization still occurs if the system is connected to many external transport channels, where uncertainties in the position of injection and exit translate into uncertainties in the spinrotation angle, unless injected electrons are prepared as spin eigenstates of the Hamiltonian. ${ }^{32}$ In Ref. 32 [see, in particular, Eq. (7) therein] spatially periodic modes in diffusive systems have been first described for the case of equal strengths $\alpha= \pm \beta$, with spatial period given by the spin-orbit length. These modes are long lived for these particular SOI strengths (and in the absence of cubic SOI) and are thus referred to as 
persistent spin helix, ${ }^{38-40}$ i.e., spin-polarization waves with specific wave vectors $\left(p_{x}, p_{y}\right)=(4 m \alpha, 0)$. The absence of spin polarization at $\alpha= \pm \beta$ for finite frequencies has been reported by Bryksin and Kleinert ${ }^{41}$ and Raichev. ${ }^{42}$

Charge currents flowing through spin-orbit-coupled diffusive metals can generate finite spin accumulations. ${ }^{14,16}$ This magnetoelectric effect achieves one of the main goals of spin orbitronics-creating a steady-state finite magnetization solely by applying an external electric field. The direction of polarization depends on the direction of the electric field and on the spin-orbit interaction. An electric field in $x$ direction leads to an accumulation in $y$ or $x$ direction for linear Rashba [Eq. (1)] or Dresselhaus [Eq. (2)] interaction, respectively. The magnetoelectric effect in presence of both Rashba and Dresselhaus interaction has been investigated in Refs. 17 and 18 which predicted that the CISP is given by the uncorrelated sum of the two accumulations generated by the Rashba and Dresselhaus SOI independent of one another. In particular, these predictions imply a finite accumulation at $\alpha= \pm \beta \neq 0$ whereas symmetry considerations (to be discussed below) require the vanishing of CISP at this point. This motivates us to revisit this issue.

The purpose of this paper is twofold. First, we revisit the Edelstein magnetoelectric effect in presence of both Rashba and Dresselhaus linear spin-orbit interactions. Contrarily to Refs. 17 and 18, we find that there is no CISP in any direction at $\alpha= \pm \beta$. However, the spin accumulation of Refs. 17 and 18 is recovered at an infinitesimally small distance away from the singular point $\alpha= \pm \beta$ in infinite systems. Our second goal is, therefore, and perhaps more importantly, to figure out to what extent phenomena occurring specifically at $\alpha= \pm \beta$ are physically relevant. To that end, we consider three possible deviations from the treatment of magnetoelectric effects given in Refs. 16-18 in the form of (i) finite-size effects, (ii) the presence of a cubic Dresselhaus interaction,

$$
H_{3 \mathrm{D}}=\gamma\left(p_{y} p_{x}^{2} \sigma_{y}-p_{x} p_{y}^{2} \sigma_{x}\right)
$$

which is always there whenever a linear Dresselhaus interaction is present, and (iii) an ac electric field. We find that spin accumulations are suppressed over parametric ranges given in each of these three instances by (i) $|\alpha|-|\beta|$ $\leqslant 1 / m L$ which depends only on the linear system size $L$, and not on the elastic mean-free path $\ell$, (ii) $|\alpha|-|\beta| \lesssim \gamma p_{\mathrm{F}}^{2}$, and (iii) $|\alpha|-|\beta| \lesssim \hbar \sqrt{\omega / m p_{\mathrm{F}} \ell}$.

There is a symmetry at $\alpha= \pm \beta$ that is responsible for the vanishing of the magnetoelectric effect. In order to expose that symmetry, we first note that a linear SOI can be considered as a nonabelian $\mathrm{SU}(2)$ gauge field with components,

$$
A_{x}=-m\left(\alpha \sigma_{y}+\beta \sigma_{x}\right), \quad A_{y}=m\left(\alpha \sigma_{x}+\beta \sigma_{y}\right), \quad A_{z}=0 .
$$

The corresponding field strength has only two nonzero components,

$$
F_{x y}=-F_{y x}=i\left[A_{x}, A_{y}\right]=-m^{2}\left(\alpha^{2}-\beta^{2}\right) \sigma_{z} .
$$

They vanish for $\alpha= \pm \beta$. Alternatively, one can consider the rotated Hamiltonian given below in Eq. (10) for $\alpha=\beta$ and perform the unitary transformation $U=e^{i \sigma_{x} \pi / 2}$ to obtain

$$
H=\left(\begin{array}{cc}
H_{+} & 0 \\
0 & H_{-}
\end{array}\right),
$$

where $H_{ \pm}=\frac{p^{2}}{2 m} \pm 2 \alpha p_{x}+V$. We thus see that the $\mathrm{SU}(2)$ gauge field reduces to two conventional $\mathrm{U}(1)$ gauge fields in the Hamiltonians $H_{ \pm}$. This $\mathrm{U}(1)$ field is a pure gauge field, implying vanishing spin conductance. To show this, one can, for instance, consider the linear-response expression for the spin conductance in a two-terminal mesoscopic sample, ${ }^{43}$

$$
G_{\mu}=\int_{C_{i}, C_{j}} d \mathbf{x} d \mathbf{x}^{\prime} \operatorname{Tr}\left[G^{R}\left(\mathbf{x}, \mathbf{x}^{\prime}\right) J_{i}^{\prime} G^{A}\left(\mathbf{x}^{\prime}, \mathbf{x}\right) J_{j}^{\mu}\right],
$$

where the trace is over the spin degree of freedom, the integrals are performed over cross sections $C_{i, j}$ of the two leads connecting the system to external terminals and the current operators $J_{i}=\left(i \nabla_{i}-\mathbf{A}\right) / m$ and $J_{j}^{\mu}=\left\{J_{j}, \sigma_{\mu}\right\}$. We write $G^{R, A}\left(\mathbf{x}, \mathbf{x}^{\prime}\right)=g^{R, A}\left(\mathbf{x}, \mathbf{x}^{\prime}\right) e^{ \pm i \mathbf{A} \cdot\left(\mathbf{x}-\mathbf{x}^{\prime}\right)}$, where $g^{R(A)}$ is the retarded (advanced) Green's function of the system in the absence of SOI. For $\alpha= \pm \beta$, one can gauge the SOI field out of the current operators via the transformation

$$
e^{i \mathbf{A} \cdot\left(\mathbf{x}-\mathbf{x}^{\prime}\right)} J_{i} e^{-i \mathbf{A} \cdot\left(\mathbf{x}-\mathbf{x}^{\prime}\right)}=\frac{i}{m} \nabla_{i},
$$

which simultaneously gauges out the spin dependence of the Green's function in Eq. (7). We thus obtain $(\mu=x, y, z)$,

$$
G_{\mu}=\int_{C_{i}, C_{j}} d \mathbf{x} d \mathbf{x}^{\prime} \nabla_{j}^{\prime} g^{R}\left(\mathbf{x}, \mathbf{x}^{\prime}\right) \nabla_{i}^{\prime} g^{A}\left(\mathbf{x}^{\prime}, \mathbf{x}\right) \operatorname{Tr}\left[\sigma_{\mu}\right]=0 .
$$

It is straightforward to see that this gauge argument also applies to CISP since the latter is given by a formula similar to Eq. (7) with the operator $J_{j}^{\mu}$ replaced by a Pauli matrix.

This paper is organized as follows. In Sec. II, we use spinand charge-coupled diffusion equations to calculate the spin accumulation generated by a charge current flowing in a bulk diffusive sample with Rashba and Dresselhaus spin-orbit interactions. This approach allows us to consider spin polarization in a finite-size system (Sec. II A), an ac external electric field (Sec. II B) and in the presence of a cubic Dresselhaus interaction (Sec. II C). Section III presents numerical results on a tight-binding Hamiltonian confirming our analytical predictions. A summary of our results and final comments are given in the conclusions section.

\section{ELECTRICALLY INDUCED SPIN POLARIZATION NEAR $\alpha= \pm \beta$}

We consider a disordered two-dimensional electron gas (2DEG) with noninteracting electrons of mass $m$ and charge $e$. Choosing coordinates $\mathbf{x}, \mathbf{y}$ and spin projections $\sigma_{x}$ and $\sigma_{y}$ along the crystal axes [1피 $]$ and [110], respectively, ${ }^{44}$ the system is described by the Hamiltonian

$$
H=\frac{p^{2}}{2 m}+\boldsymbol{\Omega}(\mathbf{p}) \cdot \sigma+V(\mathbf{x}) .
$$

Here, $\mathbf{p}=\left(p_{x}, p_{y}, 0\right)$ is the electron's momentum, $\sigma$ $=\left(\sigma_{x}, \sigma_{y}, \sigma_{z}\right)$ is a vector of Pauli matrices (we later use $\sigma^{0}$ $=1$ ), and 


$$
\boldsymbol{\Omega}(\mathbf{p})=\sum_{k, j=1}^{3} \Omega_{k j} p_{j} \mathbf{e}_{k}=\left(-(\alpha-\beta) p_{y},(\alpha+\beta) p_{x}, 0\right)
$$

is the internal field due to Rashba and linear Dresselhaus SOI given in Eqs. (1) and (2), with strength $\alpha$ and $\beta$, respectively. The disorder potential $V$ is due to static, short ranged, and randomly distributed impurities leading to a mean-free path $\ell=p_{\mathrm{F}} \tau / m$, where $\tau$ is the elastic scattering time and $p_{\mathrm{F}}$ is the Fermi momentum. The interplay of disorder and SOI is characterized by dimensionless parameters $\xi_{\alpha}=2 \alpha p_{\mathrm{F}} \tau, \xi_{\beta}$ $=2 \beta p_{\mathrm{F}} \tau(\hbar \equiv 1)$ measuring the spin-precession angle due to Rashba and Dresselhaus SOI between two consecutive scatterings at impurities. Our treatment presupposes $\xi_{\alpha, \beta} \ll 1$, which ensures that spin distribution functions vary slowly everywhere across the sample.

The coupled spin and charge excitations of the Rashba/ Dresselhaus spin-orbit-coupled 2D electron gas obey the following integral equation (summation over doubly-occurring indices is assumed):

$$
S_{\mu}(\mathbf{r}, \omega)=\int \frac{d \mathbf{r}^{\prime}}{2 m \tau} \operatorname{Tr}\left[\sigma_{\mu} G_{E}^{R}\left(\mathbf{r}, \mathbf{r}^{\prime}\right) \sigma_{\nu} G_{E-\omega}^{A}\left(\mathbf{r}^{\prime}, \mathbf{r}\right)\right] S_{\nu}\left(\mathbf{r}^{\prime}, \omega\right),
$$

where $S_{x, y, z}(\mathbf{r}, \omega)$ and $S_{0}(\mathbf{r}, \omega)=n(\mathbf{r}, \omega)$ are the spin and charge distribution functions, respectively. We obtain diffusion equations in the presence of both Dresselhaus and Rashba SOI by gradient expanding this integral equation. For $\beta=0$, these equations have been derived using diagrammatic perturbation theory, ${ }^{7}$ kinetic equations, ${ }^{6}$ and quantum Boltzmann-equation approaches. ${ }^{8}$ For finite $\alpha$ and $\beta$ we obtain the same diffusion equations as in Ref. 38 which we rewrite here for convenience

$$
\begin{gathered}
\partial_{t} n=D \nabla^{2} n+K_{s-c}^{x} \partial_{x} S_{y}-K_{s-c}^{y} \partial_{y} S_{x}, \\
\partial_{t} S_{x}=D \nabla^{2} S_{x}-K_{s-c}^{y} \partial_{y} n-K_{p}^{x} \partial_{x} S_{z}-\Gamma_{x} S_{x}, \\
\partial_{t} S_{y}=D \nabla^{2} S_{y}+K_{s-c}^{x} \partial_{x} n-K_{p}^{y} \partial_{y} S_{z}-\Gamma_{y} S_{y}, \\
\partial_{t} S_{z}=D \nabla^{2} S_{z}+K_{p}^{y} \partial_{y} S_{y}+K_{p}^{x} \partial_{x} S_{x}-\Gamma_{z} S_{z} .
\end{gathered}
$$

Here the spin-charge couplings $K_{s-c}^{x, y}$, precession couplings $K_{p}^{x, y}$, and spin-relaxation rates $\Gamma_{x, y}$ are given by

$$
\begin{gathered}
K_{s-c}^{x}=4 m^{2} D \tau(\alpha-\beta)^{2}(\alpha+\beta), \\
K_{s-c}^{y}=4 m^{2} D \tau(\alpha+\beta)^{2}(\alpha-\beta), \\
K_{p}^{x}=4 m D(\alpha+\beta), \\
K_{p}^{y}=4 m D(\alpha-\beta), \\
\Gamma_{x}=1 / \tau_{x}=4 m^{2} D(\alpha+\beta)^{2}, \\
\Gamma_{y}=1 / \tau_{y}=4 m^{2} D(\alpha-\beta)^{2}, \\
\Gamma_{z}=\Gamma_{x}+\Gamma_{y} .
\end{gathered}
$$

For a homogeneous sample with a homogeneous charge current density, it is tempting to assume homogeneous spin ac- cumulations and ignore all partial derivatives of $S_{\mu}$ to obtain

$$
\begin{gathered}
S_{x} \propto-K_{s-c}^{y} \tau_{x} \partial_{y} n=-(\alpha-\beta) \tau \partial_{y} n, \\
S_{y} \propto K_{s-c}^{x} \tau_{y} \partial_{x} n=(\alpha+\beta) \tau \partial_{x} n, \\
S_{z}=0 .
\end{gathered}
$$

for the bulk Edelstein CISP. We note the cancellation of the potentially singular $(\alpha \pm \beta)$ factors in $\tau_{x, y}$. For $\alpha \rightarrow \pm \beta$ the spin-charge couplings go to zero but this behavior seems to be canceled by the diverging spin-relaxation time to give finite spin accumulations at $\alpha= \pm \beta$. However the same approach for $\alpha$ set to $\beta$ from the outset produces vanishing spin accumulations. The main reason behind this inconsistency is that one spin-relaxation time of the system diverges as $\alpha$ $\rightarrow \pm \beta$. However, in a real finite-sized system, the spinrelaxation time is bounded from above by the typical time to escape to the leads. This is so because leads provide spin (and charge) relaxation, which for $\alpha= \pm \beta$ becomes the dominant spin-relaxation mechanism. Finite-sized effects are thus expected to induce a smooth crossover to zero CISP as $\alpha \rightarrow \pm \beta$. In the next section, we show that this is indeed the case.

\section{A. Electrically induced spin polarization in finite systems}

We assume a rectangular sample with SOI attached to two external reservoirs defining the current direction and bounded by vacuum otherwise. We obtain for the charge distribution function

$$
n(E)=2(1-x / L) \mathcal{F}(E-e V)+(2 x / L) \mathcal{F}(E),
$$

where $\mathcal{F}(E)$ is the Fermi function. The appropriate boundary conditions are that the spin accumulations vanish at the reservoirs and the normal component of the spin current vanishes at the hard wall boundaries. ${ }^{19,45}$

Solving the diffusion equations we obtain the maximum spin accumulation within the SOI region for an electric field along the $x$ direction,

$$
\begin{gathered}
S_{y}=S_{2 \mathrm{DEG}}[1-1 / \cosh (m L|\alpha-\beta| / \hbar)], \\
S_{2 \mathrm{DEG}}=(\alpha+\beta) \tau \frac{d n}{d x} .
\end{gathered}
$$

For a field in the $y$ direction, one has the same behavior for $S_{x}$ instead of $S_{y}$, with $|\alpha+\beta|$ in the argument of the cosh and $S_{2 \mathrm{DEG}}=-(\alpha-\beta) \tau d n / d y$. Equation (15) shows that the Edelstein CISP goes smoothly to zero for $\alpha= \pm \beta$, with the width of the crossover set solely by the system size, generating a singular behavior only as $L \rightarrow \infty$. The size of the crossover region is, in particular, independent of the mean-free path $\ell$, hence of the strength of the impurity potential, since in our regime, $\xi_{\alpha, \beta} \ll 1$, the spin-orbit relaxation length is independent of disorder. Away from $\alpha= \pm \beta$, one recovers the standard CISP $S_{2 \text { DEG }}$ predicted in Refs. 17 and 18 . The validity of Eq. (15) is illustrated numerically below in Fig. 2. 


\section{B. ac-field-induced spin polarization}

We next discuss the frequency dependence of CISP due to an ac electric field within the framework of the diffusion Eq. (12a). For $\alpha= \pm \beta$, this problem has already been addressed by Kleinert and Bryksin ${ }^{41}$ and Raichev, ${ }^{42}$ and we revisit it briefly only for completeness. In an infinite system the polarization is spatially homogeneous such that all derivatives of $S_{\mu}$ in Eqs. (12b)-(12d) vanish. The resulting bulk polarization then satisfies

$$
\begin{gathered}
\left(-i \omega+\Gamma_{x}\right) S_{x}=-K_{s-c}^{y} \partial_{y} n, \\
\left(-i \omega+\Gamma_{y}\right) S_{x}=K_{s-c}^{x} \partial_{x} n \\
\left(-i \omega+\Gamma_{z}\right) S_{z}=0 .
\end{gathered}
$$

Further neglecting the influence of SOI on $n$ one finds from Eq. (15) that $\nabla n=-2 \nu e \mathbf{E}$ and thus

$$
\begin{gathered}
S_{x}=2 \nu e E_{y}(\alpha-\beta) \operatorname{Re}\left[\Gamma_{x} /\left(\Gamma_{x}-i \omega\right)\right], \\
S_{y}=-2 \nu e E_{x}(\alpha+\beta) \operatorname{Re}\left[\Gamma_{y} /\left(\Gamma_{y}-i \omega\right)\right], \\
S_{z}=0
\end{gathered}
$$

for finite but small $\omega \tau \ll 1$. This result has been found in Refs. 46 and 47. As for finite-sized systems, we see that both spin accumulations vanish at $\alpha= \pm \beta$ and that the result of Refs. 17 and 18 is only recovered at $|\alpha|-|\beta| \sim \sqrt{\omega / m p_{\mathrm{F}} \ell}$. In the limit $\omega \tau \rightarrow 0$ the polarization vanishes at the singular points only.

\section{CISP in presence of a cubic Dresselhaus interaction}

A linear Dresselhaus SOI, Eq. (1), is always accompanied by a cubic Dresselhaus interaction, Eq. (3), whose strength might or might not be much weaker than that of the linear SOI. Because the presence of a cubic Dresselhaus SOI breaks $\mathrm{U}(1)$ symmetry at $\alpha= \pm \beta$, whose presence is crucial to the vanishing of the CISP, we investigate in this paragraph the effect that a cubic Dresselhaus SOI has on the CISP close to those points.

If the cubic contributions are weak we still expect a suppression of the CISP at $\alpha= \pm \beta$ and that the additional spin relaxation due to $H_{3 \mathrm{D}}$ renders the point $\alpha= \pm \beta$ nonsingular in the absence of boundary effects and at zero frequency. In the coordinates chosen in Eq. (10) the cubic term in the SOI Hamiltonian is

$$
H_{3 \mathrm{D}}=\frac{1}{2} \gamma\left(p_{y}^{2}-p_{x}^{2}\right)\left(p_{x} \sigma_{y}-p_{y} \sigma_{x}\right)
$$

which has to be incorporated into the diffusion Eqs. (12b)-(12d). The relevant relaxation rates $\Gamma_{\mu}$ and spincharge couplings $K_{s-c}^{\mu}$ have been calculated in Refs. 46 and 48 , respectively. In our notation they are given by

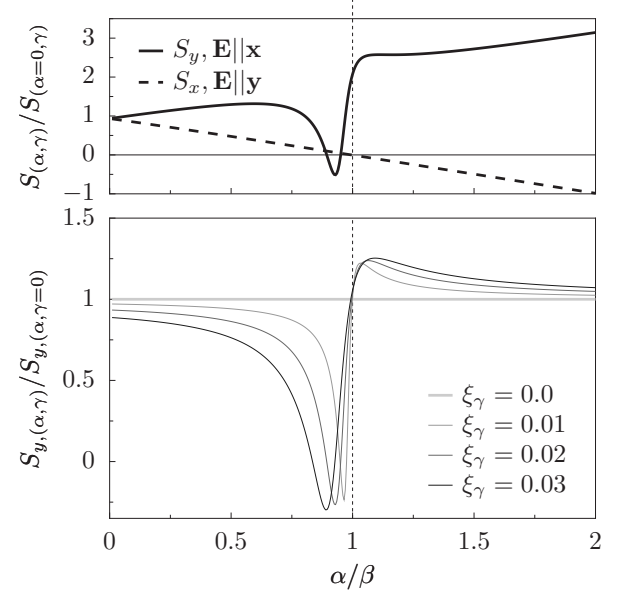

FIG. 1. Upper panel: Spin polarization $S_{x,(\alpha)} / S_{x,(\alpha=0)}$ for $\mathbf{E} \|[110]$ (dashed) and $S_{y,(\alpha)} / S_{y,(\alpha=0)}$ (solid line) for $\mathbf{E} \|[1 \overline{1} 0]$ as a function of Rashba SOI $\alpha / \beta$ for $\xi_{\beta}=2 \beta p_{\mathrm{F}} \tau=0.1$ and $\xi_{\gamma}=0.02$. Lower panel: $\alpha$ dependence of the normalized spin polarization $S_{y,(\alpha, \gamma)} / S_{y,(\alpha, \gamma=0)} \quad$ for $\quad \mathbf{E} \|[110], \quad \xi_{\beta}=2 \beta p_{\mathrm{F}} \tau=0.1, \quad$ and $\quad \xi_{\gamma}$ $=0.0,0.01,0.02,0.03$.

$$
\begin{aligned}
\Gamma_{x, y}= & \frac{\left(\xi_{\alpha} \pm \xi_{\beta}\right)^{2}}{2 \tau}-\frac{\xi_{\gamma}\left(\xi_{\beta} \pm \xi_{\alpha}\right)}{4 \tau}+\frac{\xi_{\gamma}^{2}}{16 \tau} \\
=D m^{2}\left[4(\alpha \pm \beta)^{2}-2(\beta \pm \alpha) \gamma p_{\mathrm{F}}^{2}+\frac{1}{2} \gamma^{2} p_{\mathrm{F}}^{4}\right] & \\
K_{s-c}^{x, y}= & (\alpha \pm \beta) \frac{\left(\xi_{\alpha} \mp \xi_{\beta}\right)^{2}}{2} \pm \frac{3}{4}\left(\alpha^{2}-\beta^{2}\right) \tau p_{\mathrm{F}} \xi_{\gamma} \\
& +\frac{1}{16}(3 \alpha \mp \beta) \xi_{\gamma}^{2} \pm \frac{3 \xi_{\gamma}^{3}}{256 \tau p_{\mathrm{F}}},
\end{aligned}
$$

where $\xi_{\gamma}=2 \gamma p_{\mathrm{F}}^{3} \tau$ and the upper (lower) sign applies to the $x$ (y) component. In the presence of cubic SOI the relation $K_{s-c}^{x, y}=\tau \Gamma_{y, x}(\alpha \mp \beta)$, which led to the cancellation of divergent terms in Eq. (14a)-(14c), no longer holds. The polarization is given by Eq. (17),

$$
S_{\mu}=2 \nu e \epsilon_{z \mu \nu} \Gamma_{\mu}^{-1} K_{s-c}^{\nu} E_{\nu},
$$

where now $\Gamma_{x, y}$ and $K_{s-c}^{x, y}$ are given in Eq. (20b), and $\epsilon_{z \mu \nu}$ is the totally antisymmetric tensor of order 3. The CISP is a rational function of $\xi_{\alpha, \beta, \gamma}$.

Figure 1 shows the behavior of $S_{x, y}$ in the presence of weak cubic Dresselhaus SOI $\left(\xi_{\gamma}=2 \gamma p_{\mathrm{F}}^{3} \tau \ll \xi_{\beta}\right)$, as a function of $\alpha / \beta$. In this case, the polarization $S_{y}$ does not vanish precisely at $\alpha=\beta$ but shows a feature in the vicinity of this point. The minimum and maximum around the feature are at $\alpha=\beta\left[1 \mp \xi_{\gamma} /\left(\xi_{\beta} 2 \sqrt{2}\right)\right]$. The zeros are at $\alpha=\beta\left(1-\xi_{\gamma} / 2 \xi_{\beta}\right)$ and $\alpha=\beta\left(1-\xi_{\gamma} / 4 \xi_{\beta}\right)$. Thus we conclude that a weak cubic Dresselhaus interaction regularizes the singularity of the CISP around $\alpha= \pm \beta$. The suppression of the CISP occurs over a width $\propto \gamma p_{\mathrm{F}}^{2}$ around $\alpha= \pm \beta$. The predicted analytical dependences of $S_{\mu}$ on Rashba and Dresselhaus SOI strengths in Eqs. (20a), (20b), and (21) may serve as guidance when 
attempting to tune quantum wells to the symmetry points $\alpha$ $= \pm \beta$ and demonstrate the vanishing of the CISP due to linear SOI at this point.

We briefly comment on the effect of extrinsic spin-orbit interaction. In Eq. (21) we found that the spin polarization is no longer singular at $\alpha= \pm \beta$ in the presence of cubic SOI due to an additional, nonvanishing relaxation rate in Eq. (20a). A similar regularization might be expected due to the relaxation rate caused by extrinsic SOI. However, the modification of the diffusion equations due to extrinsic SOI (Refs. 1,49 , and 50) and the resulting spin polarization are beyond the scope of the paper.

\section{NUMERICAL SIMULATIONS}

We now perform quantum transport simulations demonstrating the suppression of the CISP around the singular point $\alpha= \pm \beta$ for finite-size geometries. To this end we consider coherent electron transport in a disordered quantum wire of width $W$ with linear Rashba and Dresselhaus SOI. For the calculations we use a tight-binding version of the Hamiltonian (10) that we obtain from a discretization of the system on a square grid with lattice spacing $a$. The Hamiltonian is $H=H_{0}+H_{\text {so }}$ with

$$
\begin{gathered}
H_{0}=-t \sum_{q, \sigma}\left(c_{q, \sigma}^{\dagger} c_{q+\hat{x}, \sigma}+c_{q, \sigma}^{\dagger} c_{q+\hat{y}, \sigma}+\text { H.c. }\right)+\sum_{q, \sigma} U_{q} c_{q, \sigma}^{\dagger} c_{q, \sigma}, \\
H_{\mathrm{so}}=\sum_{q}\left[-\left(t_{\mathrm{R}}+t_{\mathrm{D}}\right)\left(c_{q, \uparrow}^{\dagger} c_{q+\hat{x}, \downarrow}-c_{q, \downarrow}^{\dagger} c_{q+\hat{x}, \uparrow}\right)+i\left(t_{\mathrm{R}}-t_{\mathrm{D}}\right)\right. \\
\left.\quad \times\left(c_{q, \uparrow}^{\dagger} c_{q+\hat{y}, \downarrow}+c_{q, \downarrow}^{\dagger} c_{q+\hat{y}, \uparrow}\right)+\text { H.c. }\right] .
\end{gathered}
$$

Here $c_{q, \sigma}^{\dagger}\left(c_{q, \sigma}\right)$ creates (annihilates) an electron with spin $\sigma=\uparrow$ or $\downarrow$ in $\hat{z}$ direction on site $q=\left(q_{x}, q_{y}\right)$. The vectors $\hat{x}$ and $\hat{y}$ have length $a$ and point in $x$ and $y$ directions, $t=1 / 2 m a^{2}$ denotes the hopping energy while $t_{\mathrm{R}}=\alpha / 2 a$ and $t_{\mathrm{D}}=\beta / 2 a$ are the Rashba and Dresselhaus SOI strength, respectively, in terms of which the spin-orbit lengths are given by $\ell_{\text {so }}^{\mathrm{R} / \mathrm{D}}$ $=\pi a t / t_{\mathrm{R}, \mathrm{D}}$. We furthermore include spin-independent disorder of Anderson type in the region of length $L$, where the on-site energies are randomly box distributed with $U_{q}$ $\in[-U / 2, U / 2]$. The disorder strength $U$ determines the elastic mean-free path $\ell \approx 48 a t^{3 / 2} \sqrt{E_{\mathrm{F}}} / U^{2}$, which we tuned to values large enough that the system is not localized but much smaller than the size of the disordered region in all our simulations.

We obtain the local electron and spin densities as

$$
\begin{aligned}
n & =-i \operatorname{Tr}\left[G^{<}(q, q)\right], \\
S_{\mu} & =-i \operatorname{Tr}\left[\sigma_{\mu} G^{<}(q, q)\right]
\end{aligned}
$$

at site $q$ by numerically computing the lesser Green's function $G^{<}(q, q)$. To this end we employ an efficient recursive lattice Green's-function method based on matrix-reordering algorithms as described in Ref. 51. We calculate averaged quantities $\left\langle S_{i}\right\rangle$ and $\langle n\rangle$, over several thousands of disorder configurations and over a rectangular region in the center of

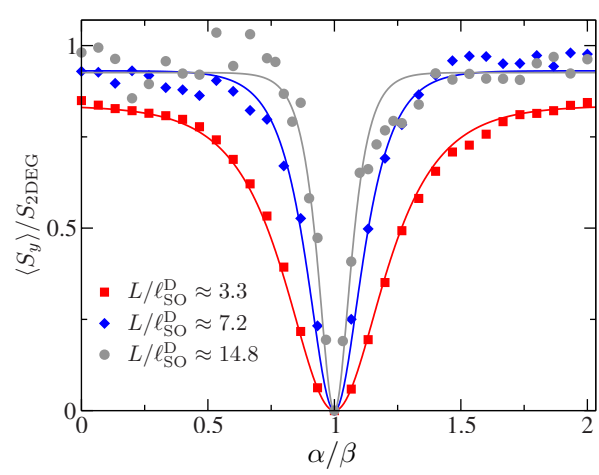

FIG. 2. (Color online) Normalized spin accumulation $S_{y} / S_{2 \mathrm{DEG}}$ as a function of $\alpha / \beta$ for fixed $\beta / 2 a=t_{\mathrm{D}}=0.15 t$ (giving $\ell_{\mathrm{so}}^{\mathrm{D}} \approx 21 a$ ), $U=2 t$ (giving $\ell \approx 8.5 a$ ), and Fermi energy $E_{\mathrm{F}}=0.5 t$, for different linear system size $W=L=70 a$ (red squares), $150 a$ (blue diamonds), and $310 a$ (gray circles). Data are averaged over 5000 disorder configurations. The solid lines are the theoretical prediction, Eq. (16), with renormalized bulk spin accumulation and system size, $S_{2 \mathrm{DEG}}$ $\rightarrow \delta_{\mathrm{fit}} S_{2 \mathrm{DEG}}$ and $L \rightarrow L_{\mathrm{fit}}$ with $\delta_{\mathrm{fit}} \approx 0.84, L_{\mathrm{fit}} \approx 39.3 a$ for $L=70 a$, $\delta_{\mathrm{fit}} \approx 0.93, L_{\mathrm{fit}} \approx 69.7 a$ for $L=150 a$, and $\delta_{\mathrm{fit}} \approx 0.93, L_{\mathrm{fit}} \approx 117.1 a$ for $L=310 a$. The electric current is in the direction $\hat{x} \|[1 \overline{1} 0]$.

the disordered part of the wire. We compare numerical data with the analytical prediction of Eq. (16). In Fig. 2 we show the normalized, spatially averaged spin accumulation, $\left\langle S_{y}\right\rangle / S_{2 \mathrm{DEG}}$, as a function of $\alpha / \beta$ varying the linear system size $L$. As expected, we find complete suppression of $\left\langle S_{y}\right\rangle$ at $\alpha=\beta$, in agreement with Eq. (16). Moreover, the pronounced dip around $\alpha=\beta$ becomes sharper and sharper as $L$ increases, and the numerical data are in good qualitative agreement with the predicted line shape, Eq. (16), in particular, they have the same parametric dependence. The agreement becomes even quantitative if one normalizes the system size and the bulk spin accumulation in Eq. (16), as is done in Fig. 2. We justify this normalization by the effective reduction in the spin-orbit interaction in confined systems with homogeneous SOI, ${ }^{52}$ and the fact that $\ell_{\text {so }}^{\mathrm{D}} \approx 2.5 \ell$ is barely in the regime of validity $\ell_{\mathrm{so}} \gg \ell$ of Eq. (16). This leads to smaller bulk spin accumulations and a longer spin-relaxation length $L_{\mathrm{s}}=\sqrt{D \tau_{x, y}}$ than the case in which the conditions $\xi_{\alpha, \beta} \ll 1$ and $L_{\mathrm{s}} \ll L$ are completely fulfilled, and qualitatively explains the renormalization of the effective system length and the bulk spin accumulation. We also note that finite-sized effects lead to deviations from our estimates $\ell \approx 48 a t^{3 / 2} \sqrt{E_{\mathrm{F}}} / U^{2}$ for the elastic mean-free path, and that numerical estimates based on the average inverse participation ratio $^{53}$ of eigenstates systematically give a larger value for $\ell$ for which $\ell / \ell_{\mathrm{so}}^{\mathrm{D}} \simeq 0.55$.

According to Eq. (16), the suppression of the CISP is independent of the strength of disorder/the elastic mean-free path of the sample, as long as one stays in the diffusive regime. This prediction is supported by our numerical calculations. We find that the spin accumulation stays approximately constant with respect to the electronic mean-free path. This is shown in Fig. 3(a). In Fig. 3(b) we moreover confirm that the CISP is independent of the width $W$ of the rectangular SOI region for $W \geq L$. However, we expect a width dependence in the form of a reduction in the CISP upon reducing $W$, when Dyakonov-Perel spin relaxation ${ }^{31}$ 

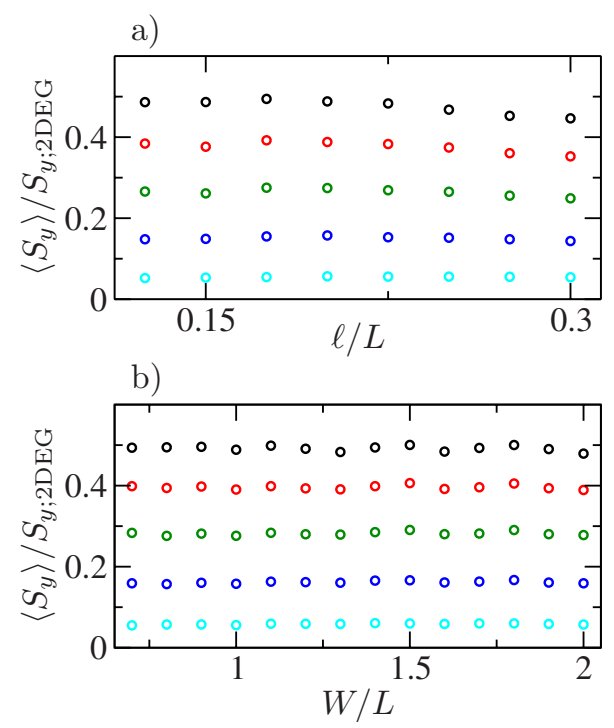

FIG. 3. (Color online) Disorder-averaged normalized spin accumulation $\left\langle S_{y}\right\rangle / S_{y ; 2 \mathrm{DEG}}$, with $S_{y ; 2 \mathrm{DEG}}=\alpha \tau(d n / d x)$, as a function of (a) the mean-free path $\ell$ (for fixed width $W=50 a$ and (b) the width $W$ of the wire (for fixed $U=2 t, \ell \approx 8.5 a$ ). The electric current is in the direction $\hat{x} \|[100]$. Different data sets correspond to different values of $\beta / \alpha=n / 15, n=10$ (black circles), 11 (red), 12 (green), 13 (dark blue), and 14 (light blue). In both panels, other parameters are fixed at $t_{\mathrm{R}}=0.15 t, E_{\mathrm{F}}=0.5 t$, and $L=40 a$ and data have been averaged over 3000 disorder configurations.

begins to be reduced and finally suppressed due to the lateral confinement. ${ }^{54,55}$

\section{CONCLUSIONS}

In this work we have studied the electrically induced and spin-orbit-mediated spin accumulation in two-dimensional diffusive conductors with emphasis on finite-size and finitefrequency effects. In the thermodynamic limit of extended systems with (linear) Rashba and Dresselhaus SOI the Edelstein magnetoelectric effect gives rise to finite spin accumulation up to suppression at the singular point $|\alpha|=|\beta|$. However, in many experimentally relevant systems, additional time, respectively, energy scales come into play, such as in tranport (i) through mesoscopic samples of finite size, (ii) in the ac regime, and (iii) through samples with cubic Dresselhaus SOI. We have shown, both analytically and numerically, that in these situations the singularity in the spin accumulation at $|\alpha|=|\beta|$ is widened to a dip. This suppression of the spin accumulation over a finite $\alpha / \beta$ range close to $\alpha= \pm \beta$ may have interesting implications with regard to other phenomena based on the Dyakonov-Perel spin-relaxation mechanism. As but one consequence, finite-size effects may render the spin-field-effect transistor proposed in Ref. 32 for $|\alpha|=|\beta|$ effectively operative even if the two linear SOI are not precisely equal. This is so because the spin rotation along two different trajectories with the same end points remains the same, even away from $|\alpha|=|\beta|$, if the trajectories are not too long. This is reflected in the finite width $|\alpha|-|\beta|$ $\lesssim 1 / m L$ of the CISP line shape given in Eq. (16). Furthermore, given that spin helices also emerge from Eqs. (12a) and (13), ${ }^{38,39}$ we conjecture that it is either finite-size effects or the presence of a cubic Dresselhaus SOI, or both, that render persistent spin helices excitable some distance away from $\alpha= \pm \beta$, and thus experimentally observable.

Recent experiments have demonstrated that GaAs quantum wells with values of $\alpha$ and $\beta$ distributed in a significantly wide range around $|\alpha|= \pm|\beta|$ can be constructed by varying their doping asymmetry and well width. ${ }^{39}$ The physics discussed above is thus of experimental relevance and can be probed as, e.g., in Refs. 56 and 57, by investigating CISP optically in several samples with different ratio $\alpha / \beta$. We predict a strong reduction in CISP in samples with $|\alpha|$ $\approx \pm|\beta|$, which can be determined independently, for instance, from the spin-helix lifetime ${ }^{39}$ or photocurrent measurements. ${ }^{58}$

While the present analysis is based on diffusive chargecarrier motion, it would be interesting to investigate ballistic mesoscopic systems and see whether our results apply there or if our analysis has to be extended. Work along these lines is in progress.

\section{ACKNOWLEDGMENTS}

We thank John Schliemann for a careful reading of the manuscript. P.J. thanks the physics department of the Universities of Basel and Geneva for their hospitality at various stages of this project and acknowledges the support of the National Science Foundation under Grant No. DMR0706319. D.L. and M.D. acknowledge financial support from the Swiss NF and the NCCR Nanoscience Basel. I.A. is supported by the funds of the Erdal Inönü Chair of Sabanci University. I.A. and K.R. thank the Deutsche Forschungsgemeinschaft for support within the cooperative research center SFB 689, and M.S. acknowledges support from the Studienstiftung des Deutschen Volkes. I.A. and P.J. express their gratitude to the Aspen Center for Physics for its hospitality.

\footnotetext{
*mathias.duckheim@unibas.ch

†matthias.scheid@uni-r.de

${ }^{1}$ M. I. Dyakonov and V. I. Perel, JETP Lett. 13, 467 (1971).

${ }^{2}$ S. Zhang, Phys. Rev. Lett. 85, 393 (2000).

${ }^{3}$ J. Sinova, D. Culcer, Q. Niu, N. A. Sinitsyn, T. Jungwirth, and A.

H. MacDonald, Phys. Rev. Lett. 92, 126603 (2004).

${ }^{4}$ S. Murakami, Phys. Rev. B 69, 241202(R) (2004).
}

${ }^{5}$ J.-I. Inoue, G. E. W. Bauer, and L. W. Molenkamp, Phys. Rev. B 70, 041303(R) (2004).

${ }^{6}$ E. G. Mishchenko, A. V. Shytov, and B. I. Halperin, Phys. Rev. Lett. 93, 226602 (2004).

${ }^{7}$ A. A. Burkov, A. S. Núñez, and A. H. MacDonald, Phys. Rev. B 70, 155308 (2004).

${ }^{8}$ I. Adagideli and G. E. W. Bauer, Phys. Rev. Lett. 95, 256602 
(2005).

${ }^{9}$ B. K. Nikolić, L. P. Zârbo, and S. Souma, Phys. Rev. B 72, 075361 (2005).

${ }^{10}$ J. Schliemann and D. Loss, Phys. Rev. B 71, 085308 (2005).

${ }^{11}$ R. Raimondi and P. Schwab, Phys. Rev. B 71, 033311 (2005).

${ }^{12}$ O. Chalaev and D. Loss, Phys. Rev. B 71, 245318 (2005).

${ }^{13}$ F. T. Vasko and N. A. Prima, Sov. Phys. Solid State 21, 994 (1979).

${ }^{14}$ L. S. Levitov, Y. V. Nazarov, and G. M. Eliashberg, Sov. Phys. JETP 61, 133 (1985).

${ }^{15}$ A. G. Aronov and Yu. B. Lyanda-Geller, JETP Lett. 50, 431 (1989).

${ }^{16}$ V. M. Edelstein, Solid State Commun. 73, 233 (1990).

${ }^{17}$ A. V. Chaplik, M. V. Entin, and L. I. Magarill, Physica E 13, 744 (2002).

${ }^{18}$ M. Trushin and J. Schliemann, Phys. Rev. B 75, 155323 (2007).

${ }^{19}$ İ. Adagideli, M. Scheid, M. Wimmer, G. E. W. Bauer, and K. Richter, New J. Phys. 9, 382 (2007).

${ }^{20}$ J. Wunderlich, B. Kaestner, J. Sinova, and T. Jungwirth, Phys. Rev. Lett. 94, 047204 (2005).

${ }^{21}$ Y. K. Kato, R. C. Myers, A. C. Gossard, and D. D. Awschalom, Science 306, 1910 (2004); V. Sih, R. C. Myers, Y. K. Kato, W. H. Lau, A. C. Gossard, and D. D. Awschalom, Nat. Phys. 1, 31 (2005).

${ }^{22}$ E. Saitoh, M. Ueda, H. Miyajima, and G. Tatara, Appl. Phys. Lett. 88, 182509 (2006); S. O. Valenzuela and M. Tinkham, Nature (London) 442, 176 (2006); T. Kimura, Y. Otani, T. Sato, S. Takahashi, and S. Maekawa, Phys. Rev. Lett. 98, 156601 (2007); 98, 249901(E) (2007).

${ }^{23}$ H. Zhao, E. J. Loren, H. M. van Driel, and A. L. Smirl, Phys. Rev. Lett. 96, 246601 (2006).

${ }^{24}$ T. Seki, Y. Hasegawa, S. Mitani, S. Takahashi, H. Imamura, S. Maekawa, J. Nitta, and K. Takanashi, Nature Mater. 7, 125 (2008).

${ }^{25}$ J. H. Bardarson, İ. Adagideli, and Ph. Jacquod, Phys. Rev. Lett. 98, 196601 (2007); İ. Adagideli, J. H. Bardarson, and Ph. Jacquod, J. Phys.: Condens. Matter 21, 155503 (2009).

${ }^{26}$ Y. V. Nazarov, New J. Phys. 9, 352 (2007).

${ }^{27}$ J. J. Krich and B. I. Halperin, Phys. Rev. B 78, 035338 (2008).

${ }^{28}$ M. Duckheim and D. Loss, Phys. Rev. Lett. 101, 226602 (2008).

${ }^{29}$ R. L. Dragomirova, L. P. Zarbo, and B. K. Nikolic, EPL 84, 37004 (2008).

${ }^{30}$ B. L. Altshuler, JETP Lett. 41, 648 (1985); P. A. Lee and A. D. Stone, Phys. Rev. Lett. 55, 1622 (1985); C. W. J. Beenakker and H. van Houten, Solid State Phys. 44, 1 (1991).

${ }^{31}$ M. I. Dyakonov and V. I. Perel, Sov. Phys. Solid State 13, 3023 (1972).

${ }^{32}$ J. Schliemann, J. C. Egues, and D. Loss, Phys. Rev. Lett. 90, 146801 (2003).

${ }^{33}$ E. I. Rashba, Sov. Phys. Solid State 2, 1109 (1960).
${ }^{34}$ G. Dresselhaus, Phys. Rev. 100, 580 (1955).

${ }^{35}$ F. G. Pikus and G. E. Pikus, Phys. Rev. B 51, 16928 (1995).

${ }^{36}$ O. Zaitsev, D. Frustaglia, and K. Richter, Phys. Rev. B 72, 155325 (2005).

${ }^{37}$ M. Scheid, M. Kohda, Y. Kunihashi, K. Richter, and J. Nitta, Phys. Rev. Lett. 101, 266401 (2008).

${ }^{38}$ B. A. Bernevig, J. Orenstein, and S.-C. Zhang, Phys. Rev. Lett. 97, 236601 (2006); P. Kleinert and V. V. Bryksin, Phys. Rev. B 76, 205326 (2007).

${ }^{39}$ J. D. Koralek, C. P. Weber, J. Orenstein, B. A. Bernevig, S.-C. Zhang, S. Mack, and D. D. Awschalom, Nature (London) 458, 610 (2009).

${ }^{40}$ M. Duckheim, D. L. Maslov, and D. Loss, Phys. Rev. B 80, 235327 (2009).

${ }^{41}$ V. V. Bryksin and P. Kleinert, Int. J. Mod. Phys. B 20, 4937 (2006).

${ }^{42}$ O. E. Raichev, Phys. Rev. B 75, 205340 (2007).

${ }^{43}$ H. U. Baranger and A. D. Stone, Phys. Rev. B 40, 8169 (1989).

${ }^{44}$ The transformation is $p \rightarrow p^{\prime}$ and $\sigma \rightarrow \sigma^{\prime}$ with $p_{x}^{\prime}=\left(p_{x}+p_{y}\right) / \sqrt{2}$, $p_{y}^{\prime}=-\left(p_{x}-p_{y}\right) / \sqrt{2}, \sigma_{x}^{\prime}=\left(\sigma_{x}+\sigma_{y}\right) / \sqrt{2}$, and $\sigma_{y}^{\prime}=-\left(\sigma_{x}-\sigma_{y}\right) / \sqrt{2}$.

${ }^{45}$ Y. Tserkovnyak, B. I. Halperin, A. A. Kovalev, and A. Brataas, Phys. Rev. B 76, 085319 (2007).

${ }^{46}$ A. G. Mal'shukov, L. Y. Wang, C. S. Chu, and K. A. Chao, Phys. Rev. Lett. 95, 146601 (2005).

${ }^{47}$ M. Duckheim and D. Loss, Phys. Rev. B 75, 201305(R) (2007).

${ }^{48}$ N. S. Averkiev, L. E. Golub, and M. Willander, J. Phys.: Condens. Matter 14, R271 (2002).

${ }^{49}$ V. L. Korenev, Phys. Rev. B 74, 041308(R) (2006).

${ }^{50}$ R. Raimondi and P. Schwab, EPL 87, 37008 (2009).

${ }^{51}$ M. Wimmer, M. Scheid, and K. Richter, in Encyclopedia of Complexity and Systems Science (Springer, New York, 2009), pp. 8597-8616; M. Wimmer and K. Richter, J. Comput. Phys. 228, 8548 (2009).

${ }^{52}$ I. L. Aleiner and V. I. Falko, Phys. Rev. Lett. 87, 256801 (2001).

${ }^{53}$ V. N. Prigodin and B. L. Altshuler, Phys. Rev. Lett. 80, 1944 (1998).

${ }^{54}$ Th. Schapers, V. A. Guzenko, M. G. Pala, U. Zulicke, M. Governale, J. Knobbe, and H. Hardtdegen, Phys. Rev. B 74, 081301(R) (2006).

${ }^{55}$ M. Scheid, I. Adagideli, J. Nitta, and K. Richter, Semicond. Sci. Technol. 24, 064005 (2009).

${ }^{56}$ Y. K. Kato, R. C. Myers, A. C. Gossard, and D. D. Awschalom, Phys. Rev. Lett. 93, 176601 (2004).

${ }^{57}$ S. D. Ganichev, S. N. Danilov, P. Schneider, V. V. Bel'kov, L. E. Golub, W. Wegscheider, D. Weiss, and W. Prettl, J. Magn. Magn. Mater. 300, 127 (2006).

${ }^{58}$ S. D. Ganichev, V. V. Bel'kov, L. E. Golub, E. L. Ivchenko, P. Schneider, S. Giglberger, J. Eroms, J. De Boeck, G. Borghs, W. Wegscheider, D. Weiss, and W. Prettl, Phys. Rev. Lett. 92, 256601 (2004). 